\title{
A transcription factor DAF-5 functions in Haemonchus contortus development
}

\author{
Wenda Di ${ }^{1,3}$, Fangfang Li ${ }^{1}$, Li He ${ }^{1,2}$, Chunqun Wang ${ }^{1}$, Caixian Zhou' ${ }^{1}$ Lu Liu' ${ }^{1}$ Lisa Ye ${ }^{1}$, Jian Chen ${ }^{1}$ and Min Hu ${ }^{1 *}$ (B)
}

\begin{abstract}
Background: Abnormal dauer formation gene (daf-5), located downstream of the DAF-7 signalling pathway, mainly functions in dauer formation and reproductive processes in the free-living nematode Caenorhabditis elegans. Although the structure and function of daf-5 have been clarified in C. elegans, they still remain totally unknown in Haemonchus contortus, a socio-economically important parasitic nematode of gastric ruminants.

Methods: A homologue of daf-5, Hc-daf-5, and its inferred product (Hc-DAF-5) in H. contortus were identified and characterized in this study. Then the transcriptional profiles of $\mathrm{Hc}$-daf-5 and the anatomical expression of Hc-DAF-5 in H. contortus were studied using an integrated molecular approach. RNA interference (RNAi) was performed to explore its function in transition from the exsheathed third-stage larvae (xL3s) to the fourth-stage larvae (L4s) in vitro. Finally, the interaction between Hc-DAF-5 and Hc-DAF-3 (a co-Smad) was detected by bimolecular fluorescence complementation (BiFc) in vitro.

Results: It was shown that Hc-DAF-5 was a member of the Sno/Ski superfamily. Hc-daf-5 was transcribed in all developmental stages of H. contortus, with significant upregulation in L3s. Native Hc-DAF-5 was localized in the reproductive organs, cuticle, and intestine via immunohistochemistry. RNAi revealed that specific small interfering RNAs (siRNAs) could retard $x$ L3 development. In addition, the interaction between Hc-DAF-5 and Hc-DAF-3 indicated that the SDS box of Hc-DAF- 5 was dispensable for the binding of Hc-DAF- 5 to Hc-DAF-3, and the MH2 domain was the binding region between Hc-DAF-3 and Hc-DAF-5.
\end{abstract}

Conclusions: In summary, these findings show that $\mathrm{Hc}$-daf-5 functions in the developmental processes of $\mathrm{H}$. contortus, and this study is the first attempt to characterize the daf-5 gene in parasitic nematodes.

Keywords: DAF-5, Development, Interaction, RNAi, siRNA, Transcription factor

\section{Background}

Transcription factors function in basal transcription by binding to particular DNA sequences in gene regulatory regions to control their transcription. Transcription factors are commonly classified into activator and repressor families based on their functions. Although the majority

\footnotetext{
*Correspondence: mhu@mail.hzau.edu.cn

1 State Key Laboratory of Agricultural Microbiology, Key Laboratory for the Development of Veterinary Products, Ministry of Agriculture, College of Veterinary Medicine, Huazhong Agricultural University, Wuhan 430070, Hubei, China

Full list of author information is available at the end of the article
}

of transcription factors have a positive effect, a number of transcription factors exert an inhibitory effect on transcription [1].

For the transforming growth factor- $\beta$ (TGF- $\beta$ ) signalling pathway in vertebrates, activation of receptors causes downstream R-Smad phosphorylation, which allows the latter to form an active Smad transcriptional complex with co-Smads. The function of this complex is affected positively or negatively by interaction with cofactor, co-activator, and co-repressor molecules, which ultimately regulates cellular processes such as proliferation, differentiation, and apoptosis [2]. As downstream components of the TGF- $\beta$ signalling pathway, the Ski original author(s) and the source, provide a link to the Creative Commons licence, and indicate if changes were made. The images or other third party material in this article are included in the article's Creative Commons licence, unless indicated otherwise in a credit line to the material. If material is not included in the article's Creative Commons licence and your intended use is not permitted by statutory regulation or exceeds the permitted use, you will need to obtain permission directly from the copyright holder. To view a copy of this licence, visit http://creativecommons.org/licenses/by/4.0/. The Creative Commons Public Domain Dedication waiver (http://creativeco mmons.org/publicdomain/zero/1.0/) applies to the data made available in this article, unless otherwise stated in a credit line to the data. 
proto-oncoprotein family of co-repressors, including c-Ski and SnoN, play important roles in tightly controlled repression of Smad-mediated transcriptional activation, and antagonize TGF- $\beta$ signalling by binding to the Smad transcriptional complex, thus preventing their interaction with co-activators, and recruiting co-repressors [3]. Additionally, Sno/Ski transcription is regulated by Smads. Thus, the degradation or accumulation of Sno/Ski protein occurs depending on the context of the TGF- $\beta$ signalling [4].

DAF-5, the homologue of vertebrate Ski/Sno in the free-living nematode Caenorhabditis elegans, functions in the TGF- $\beta$ signalling pathway (DAF-7 signalling pathway) for dauer regulation and egg-laying in this nematode, but not like a conventional Sno/Ski protein [5]. In brief, the DAF-7 signal is transduced by R-Smads. These molecules, when activated, function as a transcriptional complex and inhibit the functions of DAF-5. DAF- 5 acts as a co-factor of co-Smad (DAF-3), rather than an antagonist of DAF-3. It also acts as a complex and is antagonized by upstream R-Smad components [5].

Although the functions of Sno/Ski and DAF-5 have been clearly identified in vertebrates and C. elegans, respectively, their roles in parasitic nematodes have not yet been elaborated. Elucidating the functions of DAF-5 in parasitic nematodes will help us understand how this molecule and the DAF-7 signalling pathway work in the parasites. Herein, a homologue of $d a f-5$ was identified from Haemonchus contortus, a blood-sucking gastric parasitic nematode of small ruminants. Its temporal transcriptional profiles in different developmental stages and special expression patterns in adult worms were explored by real-time polymerase chain reaction (PCR) and immunohistochemistry, respectively. In addition, small interfering RNAs (siRNAs) were used to knock down Hc-daf-5 in exsheathed third-stage larvae (xL3s) by soaking. A bimolecular fluorescence complementation (BiFc) experiment was also performed to demonstrate the interaction between $H c$-DAF-5 and $H c$-DAF-3.

\section{Methods}

\section{Worm maintenance}

The $H$. contortus Haecon-5 strain was maintained by goats, which were infected orally with 5000 L3s. Free-living stages including eggs, first-stage larvae (L1s), secondstage larvae (L2s), and L3s were harvested from faeces of infected goats. Parasitic stages (L4s and adults) were collected from the abomasa of infected goats euthanized at 8 and 30 days, respectively, and these two developmental stages were washed extensively in $0.85 \%$ sodium chloride, and male and female worms were carefully separated under a microscope prior to storage at $-80^{\circ} \mathrm{C}$.

\section{Nucleic acid and protein preparation and gene cloning}

Total RNA was extracted from individual developmental stages of $H$. contortus using TRIzol reagent (Simgen, China). All RNA samples were treated with DNase I to remove genomic DNA (gDNA), and then their integrity and yields were verified by electrophoresis and spectrophotometric analysis. Complementary DNA (cDNA) was synthesized from RNA $(1 \mu \mathrm{g})$ using the PrimeScript RT reagent kit with gDNA Eraser (Takara, Japan) for coding sequence (CDS) amplification and real-time PCR. RNA was stored at $-80^{\circ} \mathrm{C}$ and DNA was stored at $-20^{\circ} \mathrm{C}$ until use.

The whole-worm proteins were prepared by grinding adult worms in phosphatase inhibitor and protein lysate (Roche Molecular Biochemicals, Switzerland), which were fractionated into soluble and insoluble materials after centrifugation at $10,000 \times g$ for $3 \mathrm{~min}$ at $4{ }^{\circ} \mathrm{C}$. Then the soluble material was stored at $-80^{\circ} \mathrm{C}$ with protease inhibitor (Thermo Fisher, USA) added, followed by western blot analysis.

The sequences of full-length cDNA and gDNA, were retrieved from the transcriptomic and genomic datasets of $H$. contortus. The CDS was amplified from cDNA with the primer pair Hc-daf-5-cF/Hc-daf-5-cR (Additional file 1: Table S1) using the following protocol: $98{ }^{\circ} \mathrm{C} / 10 \mathrm{~min}$, then $98{ }^{\circ} \mathrm{C} / 10 \mathrm{~s}, 55^{\circ} \mathrm{C} / 5 \mathrm{~s}$ and $72{ }^{\circ} \mathrm{C} / 2 \mathrm{~min}$ for 35 cycles, and $72{ }^{\circ} \mathrm{C} / 10 \mathrm{~min}$. The PCR product was inserted into pMD-19T and sequenced in both directions directly by Tsingke Biological Technology, China.

\section{Bioinformatics analyses}

The CDS of Hc-daf-5 were conceptually translated into predicted amino acids using DNASTAR software (http:// www.dnastar.com/). The predicted amino acid sequence of $\mathrm{Hc}$-DAF- 5 was compared with the sequences in nonredundant protein databases using BLASTP from the National Center for Biotechnology Information (NCBI) to confirm the homologous sequences.

The structural domains (Dach and SDS boxes) of $\mathrm{Hc}$ DAF-5 were aligned with its homologues from 11 species (Ancylostoma ceylanicum, Brugia malayi, Caenorhabditis briggsae, C. elegans, Danio rerio, Drosophila melanogaster, Equus caballus, Homo sapiens, Mus musculus, Nippostrongylus brasiliensis, and Toxocara canis) [6-20] (Additional file 1: Table S2) using the BioEdit program. For $\mathrm{Hc}$-DAF-5, the coiled coil region was predicted by Expasy (https://embnet.vital-it.ch/software/COILS form.html).

The full-length protein sequences from eight species (C. briggsae, C. elegans, D. rerio, D. melanogaster, $H$. sapiens, $M$. musculus, $N$. brasiliensis, and T. canis) (Additional file 1: Table S2) were aligned and used for 
phylogenetic analyses by means of the neighbour-joining $(\mathrm{NJ})$, maximum parsimony (MP), and maximum likelihood (ML) methods. Confidence limits were assessed using a bootstrap procedure employing 1000 pseudo-replicates in MEGA [21].

\section{Transcriptional analyses of $\mathrm{Hc}$-daf-5 in different stages of $H$. contortus by real-time PCR}

Real-time PCR was carried out using the specific primers Hc-daf-5-qF/Hc-daf-5-qR (Additional file 1: Table S1) to determine the mRNA levels in different developmental stages of $H$. contortus including eggs, L1s, L2s, L3s, both sexes of L4s, and adult worms. Total RNA of each stage was isolated with TRIzol reagent according to the manufacturer's instructions and treated with DNase I to remove gDNA before synthesis of cDNA. The realtime PCR conditions were set as follows: one cycle at $50{ }^{\circ} \mathrm{C} / 2$ min and $95{ }^{\circ} \mathrm{C} / 30 \mathrm{~s}, 40$ cycles at $95{ }^{\circ} \mathrm{C} / 15 \mathrm{~s}$, $60{ }^{\circ} \mathrm{C} / 15 \mathrm{~s}$, and $72{ }^{\circ} \mathrm{C} / 20 \mathrm{~s}$, and one cycle at $60{ }^{\circ} \mathrm{C} / 1 \mathrm{~min}$, $95^{\circ} \mathrm{C} / 15 \mathrm{~s}$, and $60^{\circ} \mathrm{C} / 15 \mathrm{~s}$. Each sample was tested in triplicate, with $\beta$-tubulin of $H$. contortus (GenBank: M76493) as a reference gene (using specific primers Hctubulin-qF and Hctubulin-qR; Additional file 1: Table S1), and the average threshold $(\mathrm{Ct})$ was taken to compare the relative quantities with $\operatorname{Am}(\mathrm{Am}=1)$ using the $2^{-\triangle \triangle \mathrm{Ct}}$ method [22]. This assay was carried out three times. One-way analysis of variance (ANOVA) in GraphPad Prism 6 was adopted for statistical analysis. $P$-values were calculated using the Tukey post hoc test, and $P<0.05$ was considered statistically significant.

\section{Production of polyclonal antibody against recombinant Hc-DAF-5 and immunoblot analysis}

A pair of specific primers Hc-daf-5-pF/Hc-daf-5-pR (Additional file 1: Table S1) containing double restriction sites was designed according to the CDS of Hc-daf-5, and it was employed to amplify the CDS of $\mathrm{Hc}$-daf-5 through PCR. Then the amplified sequence was cloned into the prokaryotic expression vector to create the expression plasmid pET-28a-Hc-daf-5, which was transformed into BL21 (DE3) cells of Escherichia coli, followed by $1 \mathrm{mM}$ isopropyl $\beta$-D-1-thiogalactopyranoside (IPTG) induction at $16{ }^{\circ} \mathrm{C}$ for $12 \mathrm{~h}$ to produce recombinant $\mathrm{rHc}$-DAF-5. Next, the recombinant $\mathrm{rHc}$-DAF-5 was purified using a Ni Sepharose column system (GE, USA) according to the manufacturer's protocol. Later, the purified $\mathrm{rHc}$-DAF-5 was used in immunizing New Zealand white rabbit to produce anti-Hc-DAF-5 polyclonal antibody. Finally, the titer and specificity of anti-Hc-DAF-5 polyclonal antibody were determined by enzyme-linked immunosorbent assay (ELISA) and western blot.
Western blot was performed as follows. Proteins were resolved by $12 \%$ sodium dodecyl sulfate-polyacrylamide gel electrophoresis (SDS-PAGE) and transferred onto an Immobilon ${ }^{\circledR}$-PSQ membrane (Merck Millipore Ltd.). Then the immunoblot membrane was blocked with blocking buffer $[1 \%(\mathrm{w} / \mathrm{v})$ bovine serum albumin (BSA) (BioFroxx, China)] in phosphate-buffered saline with $20 \%$ Tween-20 (PBST)] for $6 \mathrm{~h}$ at $4{ }^{\circ} \mathrm{C}$, washed three times with PBST, and incubated with $\mathrm{Hc}$-DAF-5 antiserum (1:1000 in PBST) overnight at $4{ }^{\circ} \mathrm{C}$. Next, samples were washed six times in PBST and subsequently incubated with horseradish peroxidase (HRP)-conjugated goat anti-rabbit antibody (1:1000, Beyotime Biotechnology, China) for $2 \mathrm{~h}$ at $37^{\circ} \mathrm{C}$, followed by washing an additional five times. Finally, immunodetection was performed by chemiluminescence (WesternBright ECL kit; K-12045-D10, China), and images were acquired using the ChemiDoc XRS+ system (Bio-Rad, USA).

\section{Evaluation of protein expression in $\mathrm{H}$. contortus via immunofluorescence assay}

Fresh male and female adults of $H$. contortus were fixed in $4 \%$ paraformaldehyde at $4{ }^{\circ} \mathrm{C}$ for $24 \mathrm{~h}$ and then consecutively dehydrated in an ethanol series $(75 \%$ for $4 \mathrm{~h}, 85 \%$ for $2 \mathrm{~h}, 90 \%$ for $2 \mathrm{~h}, 95 \%$ for $1 \mathrm{~h}$, and $100 \%$ for $30 \mathrm{~min}$ twice). The dehydrated worms were then incubated in xylene/absolute ethanol (1:1) solution for $5 \mathrm{~min}$ and xylene for $10 \mathrm{~min}$, and then embedded in paraffin. Next, $4 \mu \mathrm{m}$-thick paraffin-embedded sections were subjected to immunofluorescence staining. For immunofluorescence assay, the sections were treated with EDTA buffer at $100{ }^{\circ} \mathrm{C}$ for $10 \mathrm{~min}$. After blocking with $5 \%$ BSA for $20 \mathrm{~min}$, anti- $H c$-DAF-5 polyclonal antibody and goat anti-rabbit IgG antibody diluted at 1:100 were sequentially added and incubated at $4{ }^{\circ} \mathrm{C}$ overnight and at $37{ }^{\circ} \mathrm{C}$ for $50 \mathrm{~min}$. Then the sections were stained with $4^{\prime} 6$-diamidino-2-phenylindole (DAPI) for $5 \mathrm{~min}$ at $37^{\circ} \mathrm{C}$ in a dark place. After thorough washing with PBS, the sections were observed under a fluorescence microscope (Olympus CX21, Japan).

RNA interference (RNAi) in H. contortus by soaking in siRNA The CDS of Hc-daf-5 was used to design the siRNA sequences with the siRNA Design Tools program, and siRNA oligos (Additional file 1: Table S3) were chemically synthesized by Shanghai GenePharma Co., Ltd.

For RNAi, L3s were exsheathed and washed five times in $0.9 \% \mathrm{NaCl}$ solution, followed by centrifugation at $600 \times g$ with diethyl pyrocarbonate (DEPC)-treated water three times. In each silencing assay, $50 \mu \mathrm{l}$ of nematode suspension (about 5000 larvae) was placed into a 96-well plate. Then $10 \mu \mathrm{l}$ of Lipofectamine 2000 (Thermo Fisher, USA) was incubated with $5 \mu$ l of Earle's Balanced Salt 
Solution (EBSS) (pH 5.2) containing $2.5 \mu \mathrm{g} / \mu \mathrm{l}$ amphotericin, $100 \mu \mathrm{g} / \mu \mathrm{l}$ streptomycin, and $100 \mathrm{IU} / \mathrm{ml}$ penicillin (Gibco, USA) at $25^{\circ} \mathrm{C}$ for $5 \mathrm{~min}$. RNasin (0.2 U) and siRNA solutions were added for incubation for $15 \mathrm{~min}$. Three siRNAs of $\mathrm{Hc}$-daf-5 were mixed in equal amounts, and the final concentration of each siRNA was adjusted to $1 \mu \mathrm{M}$, while the final concentration of negative control siRNA was $3 \mu \mathrm{M}$, with nuclease-free water as the blank control.

The knockdown experiments were carried out as described previously [23]. In brief, three groups of xL3s (5000 in each group), which were kept in $80 \mu$ l of EBSS (pH 5.2) and supplemented with respective siRNAs, were soaked for $72 \mathrm{~h}$. Approximately 100 larvae in each group were transferred to a fresh culture medium with EBSS for another 5 days to assess their development. The remaining larvae were subjected to RNA extraction.

All RNAs were extracted using TRIzol reagent according to the manufacturer's instructions. Then cDNA was synthesized using the PrimeScript RT reagent kit with gDNA Eraser (Takara, China). Each $10 \mu \mathrm{l}$ of reaction was conducted with the same amount of cDNA from each sample. The $18 S$ gene of $H$. contortus was taken as the endogenous control using the primer pair Hc-18S-qF/ Hc-18S-qR (Additional file 1: Table S1). Primers (Hcdaf-5-qF/Hc-daf-5-qR) used in detecting transcriptional profiles were also used here for the detection of transcriptional changes of $\mathrm{Hc}$-daf-5 in worms treated with siRNA. The amplification efficiency of the primers was tested by a standard curve assay, and linear regression analysis showed similar slopes of all tested primers. The real-time PCR was performed on an ABI 7100 thermal cycler (Applied Biosystems, Germany) as per the following conditions: one cycle at $50{ }^{\circ} \mathrm{C} / 2 \mathrm{~min}$ and $95^{\circ} \mathrm{C} / 30 \mathrm{~s}$, 40 cycles at $95{ }^{\circ} \mathrm{C} / 15 \mathrm{~s}, 60^{\circ} \mathrm{C} / 15 \mathrm{~s}$, and $72{ }^{\circ} \mathrm{C} / 20 \mathrm{~s}$, and a cycle at $60^{\circ} \mathrm{C} / 1 \mathrm{~min}, 95^{\circ} \mathrm{C} / 15 \mathrm{~s}$, and $60{ }^{\circ} \mathrm{C} / 15 \mathrm{~s}$. The fold change of $\mathrm{Hc}$-daf-5 expression after RNAi was calculated by the $2^{-\Delta \Delta \mathrm{Ct}}$ method. $\Delta \Delta \mathrm{Ct}=\left[\left({ }^{\mathrm{Ct}} \mathrm{RNAi}, H c\right.\right.$-daf-5 $)-\left({ }^{\mathrm{Ct}}\right.$ RNAi, Hc-18S) $]-\left[\left({ }^{\mathrm{Ct}}\right.\right.$ Blank, Hc-daf-5) $-\left({ }^{\mathrm{Ct}}\right.$ Blank, Hc18S)] [24].

\section{Assessing the interaction between Hc-DAF- 5 and Hc-DAF-3 by bimolecular fluorescence complementation (BiFC)}

The BHK21 cells were grown and maintained in Dulbecco's modified Eagle medium (DMEM) supplemented with $10 \%$ fetal bovine serum (FBS) and 1\% penicillin/ streptomycin. The plasmids pbJun-HA-KN151 and pbFos-Myc-LC151 were used as original plasmids for plasmid construction. In brief, the CDS fragment of $\mathrm{Hc}$ daf-3 was amplified with the primer pair Hc-daf-3-fF/ Hc-daf-3-fR (Additional file 1: Table S1) and cloned into pbJun-HA-KN151 between the NheI and XhoI sites to replace bJun, thus generating the pHcDAF3-HA-KN151 plasmid. Meanwhile, the CDS fragment of $H c-d a f-5$ was amplified with the primer pair Hc-daf-5-fF/Hc-daf-5-fR (Additional file 1: Table S1) and cloned into pbFos-MycLC151 between the NheI and PvuI sites to replace bFos, so as to generate the $\mathrm{pHcDAF5-Myc-LC151} \mathrm{plasmid.} \mathrm{The}$ BHK21 cells were seeded onto six-well plates and grown to $70-80 \%$ confluence for transfection. To examine the interactions between $\mathrm{Hc}$-DAF-3 and $\mathrm{Hc}$-DAF-5, the plasmids pHcDAF3-HA-KN151 and pHcDAF5-Myc-LC151 were co-transfected using Lipofectamine 2000 (Thermo Fisher, USA). Then the cells were incubated at $37^{\circ} \mathrm{C}$ with $5 \% \mathrm{CO}_{2}$ for a further $20 \mathrm{~h}$, and fluorescence was subsequently detected at 580-680 $\mathrm{nm}$ and imaged.

In addition to the full-length constructs of $\mathrm{Hc}$-DAF-3, a DNA fragment encoding the $H c-D A F-3-M H 2$ domain (645 bp; 484-697 amino acids [aa]) was amplified with the primer pair Hc-daf-3-MH2-F/Hc-daf-3-fR (Additional file 1: Table S1), sequenced, and cloned into pHcDAF3-MH2-HA-KN151. Similarly, a DNA fragment encoding the $H c$-DAF-5-SDS domain (900 bp; 1-300 aa) was amplified with the primer pair Hc-daf-5-fF/Hc-daf5-SDS-R (Additional file 1: Table S1), sequenced, and cloned into pHcDAF5-SDS-Myc-LC151. Finally, the plasmids pbFos-Myc-LC151 and pbJun-HA-KN151, which express bJun and bFos, respectively, were co-transfected as positive controls, while pHA-KN151 and pHcDAF5SDS-Myc-LC151 were set as negative controls.

\section{Results}

\section{Sequence and phylogenetic analyses of Hc-DAF-5}

The CDS of Hc-daf-5 was 1629 bp in length (GenBank accession number: MK159305), and it was predicted to encode a protein (Hc-DAF-5) of 542 amino acids. The full-length genomic sequence of $\mathrm{Hc}$-daf-5 was $8535 \mathrm{bp}$ long, containing 11 exons (73-207 bp) and 10 introns (57-3071 bp). Compared with Ce-daf-5 [5], Hc-daf-5 had more exons and introns.

A BLAST search with the $\mathrm{Hc}$-DAF-5 protein sequences as bait against the NCBI GenBank databases revealed similarities to the SKI, SnoN, Skate, and Icy in H. sapiens and the Snowski and Iceskate in D. melanogaster (Additional file 1: Table S2) [25, 26]. The predicted protein sequence of $\mathrm{Hc}$-DAF-5 was then aligned with homologues from five nematodes and five other metazoans. Multiple sequence comparison revealed the highest identity of $\mathrm{Hc}$-DAF- 5 to SKI in $N$. brasiliensis (58.3\%), but low identity ranging from 18.7 to $28.7 \%$ to its homologues from different metazoan species, including $B$. malayi (Snowski, 22.7\%), C. briggsae (DAF-5, 21.8\%), C. elegans (DAF-5, 23.0\%), D. rerio (SKI, 24.1\%), D. melanogaster (Iceskate, 20.3\%; Snowski, 20.9\%), E. caballus (SKI, $22.9 \%$ ), H. sapiens (Icy, 18.7\%; Skate, 21.1\%; SKI, 23.1\%; 


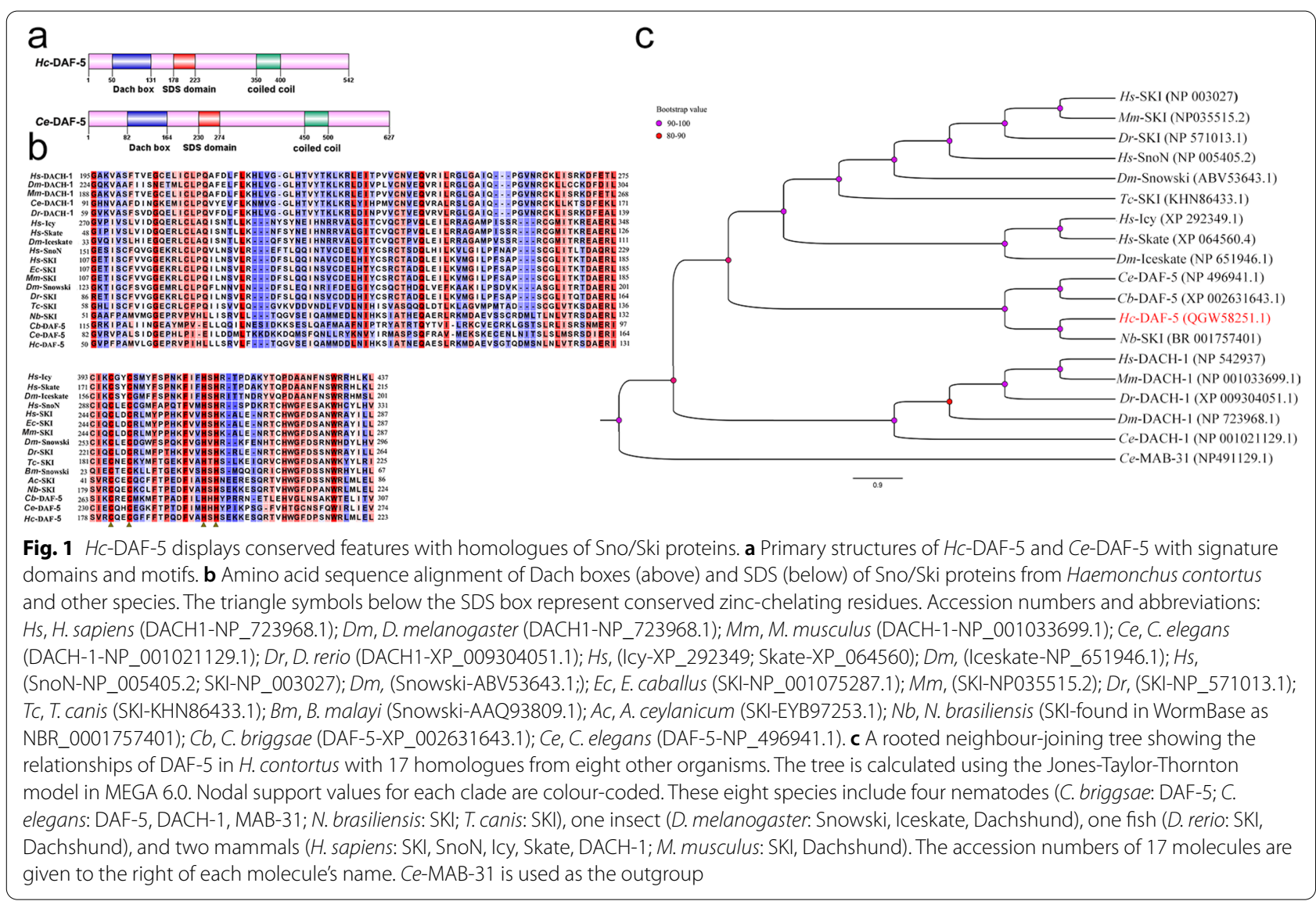

SnoN, 20.4\%), M. musculus (SKI, 21.9\%), and T. canis (SKI, 28.7\%).

The alignment of these sequences also indicated that Hc-DAF-5 had two functional domains including a Dach box (Dachshund-homology domain) and an SDS box (Smad binding domain) (Fig. 1a). Dach box is a domain shared by the Sno/Ski, Skate/Icy and Dachshund superfamily. Dachshund is a transcription regulator conserved throughout bilaterians. Alignment of the Dachshundhomology domain among selected species (Fig. 1b, above) revealed that Dach box of $\mathrm{Hc}$-DAF-5 had higher similarity to that of $\mathrm{Nb}$-SKI than to that of $\mathrm{Ce}$-DAF-5 and $C b$-DAF-5. In $H$. contortus, DAF-5 clearly possessed an SDS box in the Smad binding domain, which was not found in any other proteins in $H$. contortus (Fig. 1a). The SDS box of Hc-DAF-5 had higher similarity to that of the Snowski group than to that of the Iceskate group. In human SKI, the SDS box and about 20 amino acids on either side constituted the minimal region required for Smad4 binding, so this region was also used for alignment (Fig. 1b below). All members of this family had a conserved zinc finger in the SDS box. The SDS box of $\mathrm{Hc}$-DAF-5 had higher similarity to that of parasitic nematodes (such as B. malayi) than to that of C. elegans
(Fig. 1b, below). As with Ce-DAF-5, Hc-DAF-5 also had a predicted coiled coil structure at the C-terminus (Fig. 1a).

The results of phylogenetic analysis showed that the topologies of the MP, ML, and NJ trees were concordant. Hc-DAF-5 grouped with $N b$-SKI with $100 \%$ bootstrap support, and then they grouped with DAF-5 from $C$. elegans and C. briggsae with $92 \%$ bootstrap support. This DAF-5 cluster grouped with Ski/Sno and Ice/Skate from $H$. sapiens, $M$. musculus, D. rerio, D. melanogaster, and $T$. canis, with $85 \%$ bootstrap support. The Dachshund subfamily from C. elegans, D. melanogaster, D. rerio, $H$. sapiens, and $M$. musculus constituted an independent cluster with $99 \%$ bootstrap support (Fig. 1c).

\section{Transcription of $\mathrm{Hc}$-daf-5 and expression of $\mathrm{Hc}$-DAF-5 in $\mathrm{H}$. contortus}

The transcriptional profile of $\mathrm{Hc}$-daf-5 was explored in eight developmental stages and sexes including egg, L1, L2, L3, L4 female, L4 male, adult female, and adult male throughout the life cycle of $H$. contortus. Hc-daf-5 was transcribed at a detectable level in all tested stages and sexes, with significant upregulation in L3 (ANOVA, $F_{(7}$, 16) $=12.03$, L3 vs egg, L1, L2, L4f, L4m, and Af, $P \leq 0.0001$. L3 vs Am, $P=0.001$ ) (Fig. 2a). 


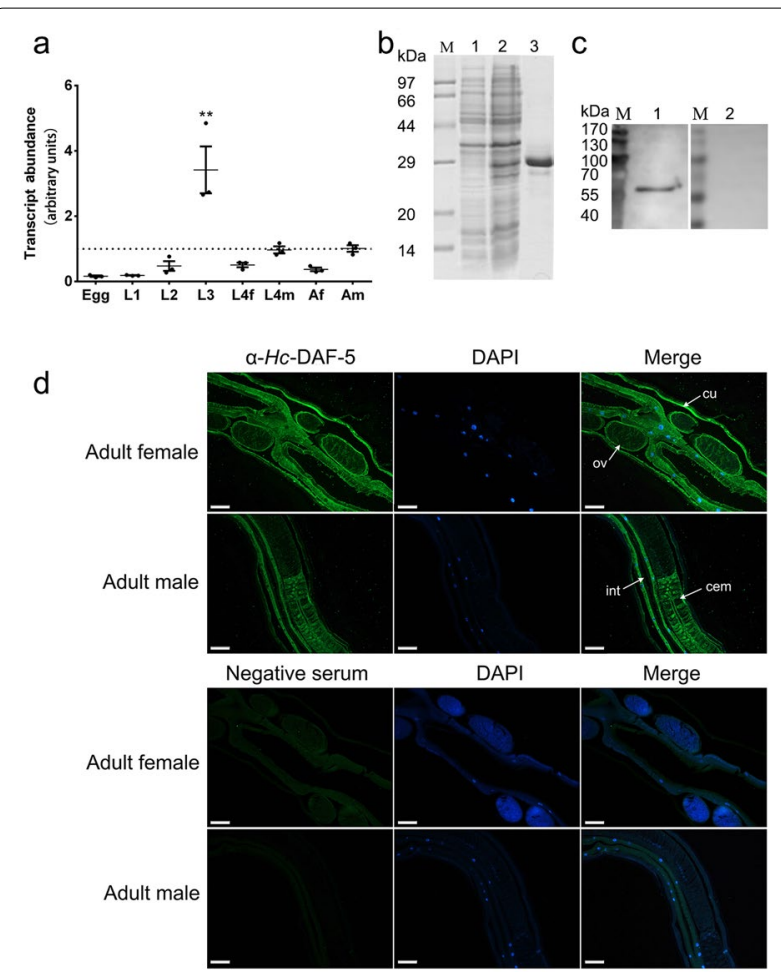

Fig. 2 Spatiotemporal expression of Hc-DAF-5 in H. contortus. a Transcriptional levels of Hc-daf-5 in different developmental stages of $\mathrm{H}$. contortus. Transcript abundance is detected and compared among eight developmental stages including eggs (Egg), the first-stage larvae (L1), the second-stage larvae (L2), the third-stage larvae $(L 3)$, the fourth-stage female $(L 4 f)$, the fourth-stage male $(L 4 m)$, adult female (Af), and adult male (Am) of $H$. contortus. The relative quantities (compared with $\mathrm{Am}, \mathrm{Am}=1$, the dotted horizontal line) are shown. All gene transcription levels are normalized to that of the $\beta$-tubulin gene. Data are presented as mean \pm SEM from three independent experiments. Significant difference is indicated by ** $(P<0.01)$. b SDS-PAGE analyses of recombinant Hc-DAF-5. Lane M: standard protein molecular weight marker. Expression of Hc-DAF-5 is induced with $1 \mathrm{mM}$ IPTG. Lane 1: recombinant Hc-DAF-5 expressed in E. coli before induction. Lane 2: recombinant Hc-DAF-5 expressed after induction. Lane 3: purified recombinant Hc-DAF-5. c Western blot analyses using total $H$. contortus protein. Lane 1: detection of Hc-DAF- 5 by the rabbit antiserum containing anti-rHc-DAF- 5 antibody. Lane 2: the same blot re-probed with pro-immune serum (as control). $\mathbf{d}$ Localization of Hc-DAF-5 in adult worms. Images in line 1 and line 2 are taken under UV light corresponding to green fluorescent protein (GFP) and DAPI, and merged images are given in line 3. Scale bars: $100 \mu \mathrm{m}$. cu (cuticle), ov (ovary), int (intestine), cem (cement gland)

Truncated Hc-DAF-5 (108-297 aa) was expressed in E. coil BL21 (DE3) with a size of $27.5 \mathrm{kDa}$ (Fig. 2b). The anti-rHc-DAF-5 polyclonal antibody was used in western blot, and a band of around $60 \mathrm{kDa}$ in total protein extracted from adult worms was recognized, while no band was recognized by the negative serum (Fig. 2c). In further immunofluorescence assay, the anti-rHc-DAF-5 polyclonal antibody detected the endogenous $H c$-DAF-5 expression in the cuticle and intestine of both female and male adult worms, as well as in the ovary of female adult worms and the cement gland of male adult worms. No fluorescence was observed in worm sections probed with the negative serum (Fig. 2d).

\section{Effect of specific siRNA on xL3 development of $\boldsymbol{H}$. contortus} As observed in previous studies, the development of $H$. contortus from xL3 to L4 underwent six stages of morphological changes, namely stage A to stage $F[27,28]$. In RNAi assay herein, the soaking of xL3s in Hc-daf-5-specific siRNA for $72 \mathrm{~h}$ resulted in a significant reduction in Hc-daf-5 transcript abundance, whereas no reduction in Hc-daf-5 transcript abundance was detected in the control siRNA-treated group or untreated group (ANOVA, $F_{(2,6)}=16.57, H c$-daf-5 siRNA-treated group versus control siRNA group, versus blank group, both $P=0.0060$ ) (Fig. 3a). In addition, the proportion of xL3s developed to stage $\mathrm{B}$ and beyond in the Hc-daf-5 siRNA-treated group was significantly lower than that in the control siRNA-treated group and untreated group (ANOVA, $\left(F_{(2,6)}=23.65\right.$, Hc-daf-5 siRNA-treated group versus control siRNA group, versus blank group, $P=0.0054$ and $P=0.0015$, respectively) (Fig. $3 \mathrm{~b}$ ).

\section{Hc-DAF-5 interacts with co-Smad Hc-DAF-3}

BiFC was employed to detect the interaction between Hc-DAF-5 and Hc-DAF-3 in live mammalian cells. The cells co-transfected with plasmid constructs as the positive controls encoding bJun-HA-KN151 and bFos-MycLC151 showed bright red fluorescence, consistent with the result in a previous report (Fig. 3c) [29]. No red fluorescence was observed in cells harbouring HA-KN151 and SDS of Hc-DAF-5 (HcDAF5-SDS-Myc-LC151) protein pair, or in the blank control (not shown). In two experimental groups, red fluorescence was observed in cells harbouring full-length $H c$-DAF-3 ( $H c$ DAF3-HAKN151) and SDS of $H c-D A F-5$ as well as in cells harbouring MH2 of Hc-DAF-3 (HcDAF3-MH2-HA-KN151) and SDS of Hc-DAF-5. However, the cells harbouring fulllength $H c$-DAF-3 and full-length $H c$-DAF-5 (HcDAF5Myc-LC151) protein pair, or MH2 of Hc-DAF-3 and full-length $\mathrm{Hc}$-DAF-5 protein pair displayed no red fluorescence (not shown).

\section{Discussion}

In the present study, $\mathrm{Hc}$-daf-5, a homologue of Ce-daf-5 and a transcription factor-encoding gene, was identified and characterized in $H$. contortus. Sequence analysis revealed that the predicted protein $\mathrm{Hc}$-DAF-5 contained a Dach box, an SDS box, and a coiled coil motif. Approximately $2-3 \%$ of all protein residues form a coiled coil 

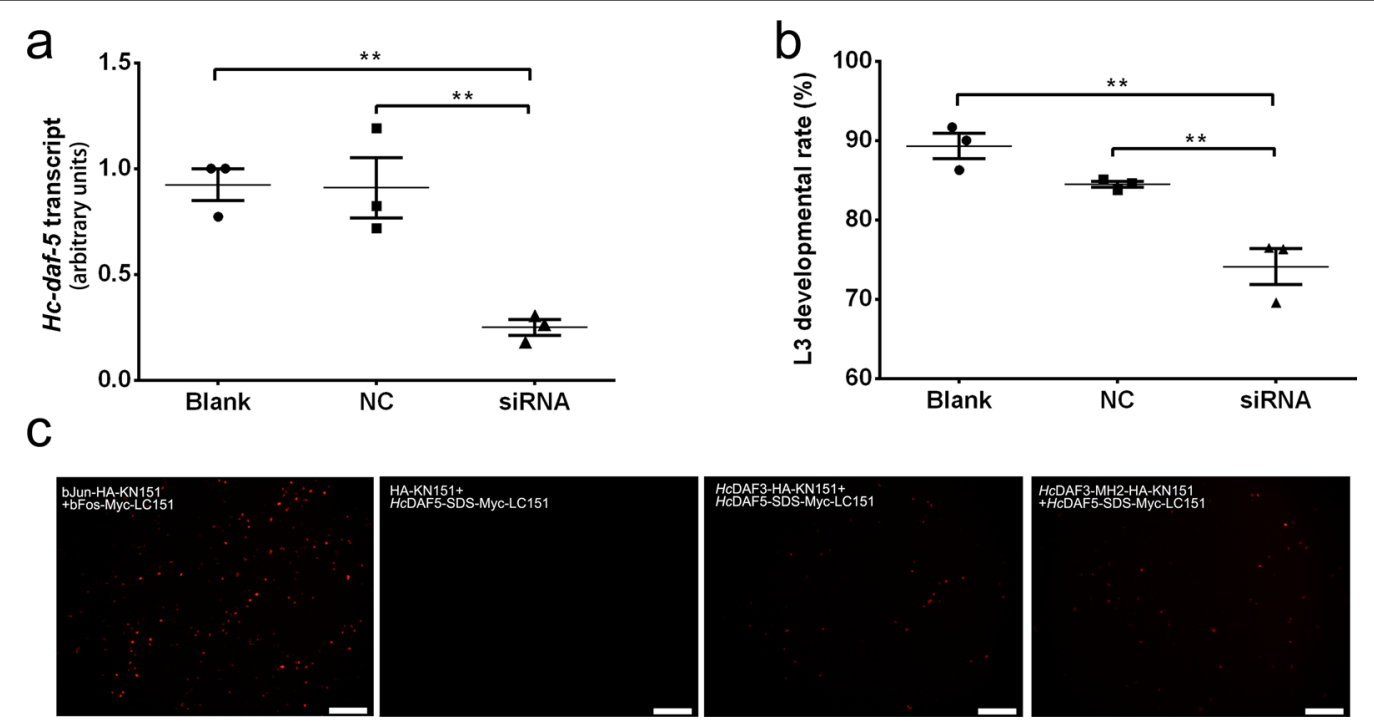

Fig. 3 Hc-DAF-5 is involved in differentiation from $x L 3$ to $L 4$ and interacts with Hc-DAF-3 in H. contortus. $\mathbf{a}$, $\mathbf{b}$ The effects of RNAi on the development of $\mathrm{H}$. contortus $\times \mathrm{L} 3$ in vitro. a The transcriptional changes of Hc-daf-5 in $\mathrm{H}$. contortus after soaking for $72 \mathrm{~h}$, detected by real-time PCR. b $L 3$ developmental rate in vitro for 5 days following RNAi. ${ }^{*} P<0.05 ;{ }^{* *} P<0.01$; ${ }^{* *} P<0.001$. Graphs in panels $\mathbf{a}$ and $\mathbf{b}$ show mean \pm SEM from three independent experiments. c Assessment of the interaction between Hc-DAF-3 and Hc-DAF-5 in live mammalian cells using BiFC. BHK21 cells are co-transfected with plasmids, and images are acquired at $20 \mathrm{~h}$ after transfection. Scale bars: $100 \mu \mathrm{m}$. Full-length Hc-DAF-3 interacts with the SDS box of HC-DAF-5 (HCDAF3-HA-KN151 and HCDAF5-SDS-Myc-LC151), while the MH2 domain of HC-DAF-3 also interacts with the SDS box of Hc-DAF-5 (HCDAF3-MH2-HA-KN151 and HCDAF-5-SDS-Myc-LC151). Positive controls (bJun-HA-KN151 and bFos-Myc-LC151) and negative controls (HA-KN151 and HCDAF5-SDSMyc-LC151) are also shown

motif, and it functions by mediating oligomerization of a large number of protein subunits. It can participate in signal-transducing events or act as a molecular recognition system [30]. The conservation of the coiled coil motif between $\mathrm{Hc}$-DAF-5 and $\mathrm{Ce}$-DAF-5 indicates the potential function of $H c$-DAF-5 in transcriptional regulation [30].

Recent research has shown that the insulin-like signalling pathway and dafachronic acid (DA) signalling pathway play a conserved role in larval development in both free-living and parasitic species [31-34]. In contrast, the role of the TGF- $\beta$ signalling pathway does not seem to be conserved between free-living and parasitic species in larval development regulation [35]. In C. elegans, Cedaf-5 is transcribed in all developmental stages including dauer larvae. Its level peaks in L1s and decreases thereafter (https://wormbase.org). However, in Strongyloides stercoralis, the transcription of Ss-daf-5 is higher in infective third-stage larvae (L3i) [36]. In the present study, the transcription of $\mathrm{Hc}$-daf-5 remained low in L1s and L2s, but peaked in L3i, which is different from that of $C e-d a f-5$ but similar to that of $S s-d a f-5$. In addition, the transcription pattern of $H c$-daf-5 is consistent with that of genes of the DAF-7 signalling pathway in $H$. contortus, including Hc-daf-3 and Hc-tgfbr-2 [37, 38]. The difference in transcriptional profiles of daf-5 between $C$. elegans and parasitic nematodes (including $H$. contortus in clade V and S. stercoralis in clade IV herein), together with that in previous studies [38], indicates divergent functions of the TGF- $\beta$ signalling pathway in free-living and parasitic species, suggesting that parasitic nematodes utilize the existing signalling pathway for different purposes in evolution.

Considering the high transcription level of $\mathrm{Hc}$-daf-5 in L3s, siRNA-mediated RNAi was performed to explore the role of $\mathrm{Hc}$-daf-5 in transition from the free-living stage (xL3) to the parasitic stage (L4). In this study, silencing of Hc-daf-5 decreased the developmental rate of $x$ L3. The experimental group treated with specific siRNAs showed fewer developed worms because the buccal development failed to initiate. This is the same effect as described for the knockdown of Hc-daf-3 and Hc-akt-1 [38, 39], and suggests that Hc-daf-5 functions in the development from xL3 to L4.

Based on the high abundance of Hc-daf-5 in L3s and its functions in xL3 development, $\mathrm{Hc}$-DAF-5 localization in L3s was attempted but failed in this study. Therefore, protein localization was performed on adult worms. Herein, native $\mathrm{Hc}$-DAF-5 protein was expressed prominently in the reproductive organs, which is consistent with $\mathrm{Hc}$-DAF-3 [38]. Considering that $\mathrm{Ce}$-DAF-5 is required for egg-laying [5], it was proposed that $\mathrm{Hc}$ DAF-5 may also function in embryonic development 
and spermatogenesis. In addition to the gonad organs, $\mathrm{Hc}$-DAF-5 was also expressed in the cuticle and intestine of adult worms. In $H$. contortus, the cuticle enables the worms to be resistant to harmful substances [40]. In summary, Hc-DAF-5 may also be associated with such processes as embryonic development and environmental resistance.

Phylogenetic analysis in this study revealed a relatively close relationship between DAF-5s in the free-living nematodes and parasitic species. In C. elegans, DAF-5 functions as a co-factor of DAF-3 (co-Smad), rather than an antagonist. However, its homologue functions as an antagonist of co-Smad in vertebrates [3]. The relatively close relationship between $\mathrm{Hc}$-DAF- 5 and $\mathrm{Ce}$-DAF-5 suggested that $H c$-DAF-5 probably also functions as a co-factor of $\mathrm{Hc}$-DAF-3. In order to test this hypothesis, $\mathrm{BiFc}$ was used for verifying the interaction between $\mathrm{Hc}$-DAF-5 and Hc-DAF-3. The results showed that the region downstream of the SDS box of $\mathrm{Hc}$-DAF- 5 was dispensable for $\mathrm{Hc}$-DAF-3 binding, and the $\mathrm{MH} 2$ domain of $\mathrm{Hc}$-DAF-3 was sufficient for its interaction with $\mathrm{Hc}$ DAF-5, consistent with the findings in C. elegans and vertebrates [5], suggesting that $\mathrm{Hc}$-DAF-3 and $\mathrm{Hc}$-DAF-5 interact with each other and function as a transcriptional regulatory complex to further regulate the downstream transcription.

\section{Conclusions}

In conclusion, a transcription factor $H c$-daf-5 in $H$. contortus was investigated. This gene was transcribed throughout the life cycle of this parasitic nematode, with the highest level in L3s, and the native $\mathrm{Hc}$-DAF-5 was expressed in the reproductive organs, cuticle, and intestine of adult worms. In addition, knockdown of $\mathrm{Hc}$-daf-5 retarded larval development in vitro. Moreover, the interaction between $\mathrm{Hc}$-DAF- 5 and $\mathrm{Hc}$-DAF-3 was also verified. In conclusion, these results provide evidence that Hc-daf-5 plays an important role in the development of H. contortus.

\section{Supplementary Information}

The online version contains supplementary material available at https://doi. org/10.1186/s13071-021-05036-2.

Additional file 1: Table S1. PCR Primers used in the present study. Table S2. Sequences of DAF-5 homologues used for alignment and phylogenetic analyses. Table S3. Sequences of Hc-daf-5-specific siRNA and control siRNA used in RNAi.

Authors' contributions

$\mathrm{MH}$ conceived the project. WDD carried out laboratory work. FFL, LH, CQW and CXZ performed the bioinformatics data analyses. LL, LSY and JC managed sheep hosts and $\mathrm{H}$. contortus isolates. WDD wrote the manuscript. $\mathrm{MH}$ received the manuscript and contributed to the final submission. All authors read and approved the final manuscript.

\section{Funding}

The authors thank the National Key Basic Research Program (973 Program) of China (Grant No. 2015CB150300), the National Natural Science Foundation (Grant No. 31872462) and the Natural Science Foundation of Hubei Province (Grant No. 2017CFA020).

\section{Availability of data and materials}

The data supporting the conclusions of this article are provided within the article.

\section{Declarations}

\section{Ethics approval and consent to participate}

Helminth-free goats were maintained in accordance with protocols approved by the Scientific Ethic Committee of Huazhong Agricultural University (Permit code: HZAUGO-2016-007) and Animal Ethics Guidelines from the People's Republic of China.

\section{Consent for publication}

Not applicable.

\section{Competing interests}

Not applicable.

\section{Author details}

${ }^{1}$ State Key Laboratory of Agricultural Microbiology, Key Laboratory for the Development of Veterinary Products, Ministry of Agriculture, College of Veterinary Medicine, Huazhong Agricultural University, Wuhan 430070 , Hubei, China. ${ }^{2}$ School of Basic Medical Sciences, Hubei University of Medicine, Hubei 442000, Shiyan, China. ${ }^{3}$ College of Animal Science and Technology, Guangxi University, Guangxi 530004, Nanning, China.

Received: 30 July 2021 Accepted: 23 September 2021

Published online: 12 October 2021

\section{References}

1. Latchman DS. Transcription factors: an overview. Int J Exp Pathol. 1993;74:417-22.

2. Liu X, Sun Y, Weinberg RA, Lodish HF. Ski/Sno and TGF-beta signaling. Cytokine Growth Factor Rev. 2001;12:1-8.

3. Frederick JP, Wang XF. Smads "freeze" when they ski. Structure. 2002;10:1607-11.

4. Stroschein SL, Wang W, Zhou S, Zhou Q, Luo K. Negative feedback regulation of TGF-beta signaling by the $\mathrm{SnoN}$ oncoprotein. Science. 1999;286:771-4.

5. da Graca LS, Zimmerman KK, Mitchell MC, Kozhan-Gorodetska M Sekiewicz K, Morales Y, et al. DAF-5 is a Ski oncoprotein homolog that functions in a neuronal TGF beta pathway to regulate $C$. elegans dauer development. Development. 2004;131:435-46.

6. Schwarz EM, Hu Y, Antoshechkin I, Miller MM, Sternberg PW, Aroian RV. The genome and transcriptome of the zoonotic hookworm Ancylostoma ceylanicum identify infection-specific gene families. Nat Genet. 2015;47:416-22.

7. Stein LD, Bao Z, Blasiar D, Blumenthal T, Brent MR, Chen N, et al. The genome sequence of Caenorhabditis briggsae: a platform for comparative genomics. PLoS Biol. 2003;1:E45.

8. Bieri T, Blasiar D, Ozersky P, Antoshechkin I, Bastiani C, Canaran P, et al. WormBase: new content and better access. Nucleic Acids Res. 2007:35:D506-10.

9. Consortium CeS. Genome sequence of the nematode C. elegans: a platform for investigating biology. Science. 1998;282:2012-8.

10. Doyle AJ, Doyle JJ, Bessling SL, Maragh S, Lindsay ME, Schepers D, et al. Mutations in the TGF-beta repressor SKI cause Shprintzen-Goldberg syndrome with aortic aneurysm. Nat Genet. 2012;44:1249-54. 
11. Pasquier J, Cabau C, Nguyen T, Jouanno E, Severac D, Braasch I, et al. Gene evolution and gene expression after whole genome duplication in fish: the PhyloFish database. BMC Genomics. 2016;17:368.

12. Hoskins RA, Carlson JW, Kennedy C, Acevedo D, Evans-Holm M, Frise E, et al. Sequence finishing and mapping of Drosophila melanogaster heterochromatin. Science. 2007;316:1625-8.

13. Matthews BB, Dos Santos G, Crosby MA, Emmert DB, St Pierre SE, Gramates LS, et al. Gene model annotations for Drosophila melanogaster: impact of high-throughput data. G3. 2015:5:1721-36.

14. Hestand MS, Kalbfleisch TS, Coleman SJ, Zeng Z, Liu J, Orlando L, et al. Annotation of the protein coding regions of the equine genome. PLoS ONE. 2015;10:e0124375.

15. Kinugawa $Y$, Uehara T, Matsuda K, Kobayashi Y, Nakajima T, Hamano H, et al. Promoter hypomethylation of SKI in autoimmune pancreatitis. Pathol Res Pract. 2018;214:492-7.

16. Xu H, Yu S, Yuan X, Xiong J, Kuang D, Pestell RG, et al. DACH1 suppresses breast cancer as a negative regulator of CD44. Sci Rep. 2017;7:4361.

17. Zhang S, Takaku M, Zou L, Gu AD, Chou WC, Zhang G, et al. Reversing SKI-SMAD4-mediated suppression is essential for TH17 cell differentiation. Nature. 2017:551:105-9.

18. Bonet F, Pereira PNG, Bover O, Marques S, Inacio JM, Belo JA. CCBE1 is required for coronary vessel development and proper coronary artery stem formation in the mouse heart. Dev Dyn. 2018;247:1135-45.

19. Eccles D, Chandler J, Camberis M, Henrissat B, Koren S, Le Gros G, et al. De novo assembly of the complex genome of Nippostrongylus brasiliensis using MinION long reads. BMC Biol. 2018;16:6.

20. Zhu XQ, Korhonen PK, Cai H, Young ND, Nejsum P, von Samson-Himmelstjerna G, et al. Genetic blueprint of the zoonotic pathogen Toxocara canis. Nat Commun. 2015;6:6145.

21. Tamura K, Stecher G, Peterson D, Filipski A, Kumar S. MEGA6: molecular evolutionary genetics analysis version 6.0. Mol Biol Evol. 2013;30:2725-9.

22. Pfaff MW. A new mathematical model for relative quantification in realtime RT-PCR. Nucleic Acids Res. 2001;29:e45.

23. He L, Gasser RB, Korhonen PK, Di W, Li F, Zhang H, et al. A TGF-beta type receptor-like molecule with a key functional role in Haemonchus contortus development. Int J Parasitol. 2018:48:1023-33.

24. Livak KJ, Schmittgen TD. Analysis of relative gene expression data using real-time quantitative PCR and the 2(-Delta Delta $C(T)$ ) Method. Methods. 2001;25:402-8.

25. Xu H, Sun F, Li X, Sun L. Down-regulation of miR-23a inhibits high glucose-induced EMT and renal fibrogenesis by up-regulation of $\mathrm{SnoN}$. Hum Cell. 2018;31:22-32.

26. Adams MD, Celniker SE, Holt RA, Evans CA, Gocayne JD, Amanatides $P G$, et al. The genome sequence of Drosophila melanogaster. Science. 2000;287:2185-95.
27. Sommerville RI. The development of Haemonchus contortus to the fourth stage in vitro. J Parasitol. 1966;52:127-36.

28. Mapes CJ. The development of Haemonchus contortus in vitro. I. The effect of $\mathrm{pH}$ and $\mathrm{pCO} 2$ on the rate of development to the fourth-stage larva. Parasitology. 1969;59:215-31.

29. Wang Y, Fang R, Yuan Y, Pan M, Hu M, Zhou Y, et al. Identification of host proteins, Spata3 and Dkk2, interacting with Toxoplasma gondii micronemal protein MIC3. Parasitol Res. 2016;115:2825-35.

30. Burkhard P, Stetefeld J, Strelkov SV. Coiled coils: a highly versatile protein folding motif. Trends Cell Biol. 2001;11:82-8.

31. Crook M. The dauer hypothesis and the evolution of parasitism: 20 years on and still going strong. Int J Parasitol. 2014;44:1-8.

32. Stoltzfus JD, Bart SM, Lok JB. CGMP and NHR signaling co-regulate expression of insulin-like peptides and developmental activation of infective larvae in Strongyloides stercoralis. PLoS Pathog. 2014;10:e1004235.

33. Albarqi MM, Stoltzfus JD, Pilgrim AA, Nolan TJ, Wang Z, Kliewer SA, et al. Regulation of life cycle checkpoints and developmental activation of infective larvae in Strongyloides stercoralis by dafachronic scid. PLoS Pathog. 2016;12:e1005358.

34. Ma G, Wang T, Korhonen PK, Young ND, Nie S, Ang CS, et al. Dafachronic acid promotes larval development in Haemonchus contortus by modulating dauer signalling and lipid metabolism. PLoS Pathog. 2019;15:e1007960.

35. Viney ME. How did parasitic worms evolve? BioEssays. 2009;31:496-9.

36. Stoltzfus JD, Minot S, Berriman M, Nolan TJ, Lok JB. RNAseq analysis of the parasitic nematode Strongyloides stercoralis reveals divergent regulation of canonical dauer pathways. PLoS Negl Trop Dis. 2012:6:e1854.

37. He L, Gasser RB, Li T, Di W, Li F, Zhang H, et al. A TGF-beta type II receptor that associates with developmental transition in Haemonchus contortus in vitro. PLoS Negl Trop Dis. 2019;13:e0007913.

38. Di W, Liu L, Zhang T, Li F, He L, Wang C, et al. A DAF-3 co-Smad molecule functions in Haemonchus contortus development. Parasit Vectors. 2019;12:609.

39. Di W, Gasser RB, He L, Li F, Liu X, Zhou C, et al. A serine/threonine-specific protein kinase of Haemonchus contortus with a role in the development. FASEB J. 2020;34:2075-86.

40. Sood ML, Kalra S. Histochemical studies on the body wall of nematodes: Haemonchus contortus (Rud., 1803) and Xiphinema insigne Loos, 1949. Z Parasitenkd. 1977;51:265-73.

\section{Publisher's Note}

Springer Nature remains neutral with regard to jurisdictional claims in published maps and institutional affiliations.
Ready to submit your research? Choose BMC and benefit from:

- fast, convenient online submission

- thorough peer review by experienced researchers in your field

- rapid publication on acceptance

- support for research data, including large and complex data types

- gold Open Access which fosters wider collaboration and increased citations

- maximum visibility for your research: over 100M website views per year

At BMC, research is always in progress.

Learn more biomedcentral.com/submissions 